PAPER

\title{
Saccadic visual search training: a treatment for patients with homonymous hemianopia
}

\author{
A L M Pambakian, S K Mannan, T L Hodgson, C Kennard
}

J Neurol Neurosurg Psychiatry 2004;75:1443-1448. doi: 10.1136/jnnp.2003.025957

See end of article for authors' affiliations

.......................

Correspondence to: A L M Pambakian, a.pambakian@ic.ac.uk

Received 14 August 2003 In revised form

23 December 2003

Accepted 16 January 2004
Objectives: We describe a novel rehabilitation tool for patients with homonymous hemianopia based on a visual search (VS) paradigm that is portable, inexpensive, and easy to deploy. We hypothesised that by training patients to improve the efficiency of eye movements made in their blind field their disability would be alleviated.

Methods: Twenty nine patients with homonymous visual field defects (HVFD) without neglect practised VS paradigms in 20 daily sessions over one month. Search fields comprising randomly positioned target and distracter elements, differing by a single feature, were displayed for three seconds on a dedicated television monitor in the patients' homes.

Improvements were assessed by examining response time (RT), error rates in VS, perimetric visual fields (VFs) and visual search fields (VSFs), before and after treatment. Functional improvements were measured using objective visual tasks which represented activities of daily living (ADL) and a subjective questionnaire.

Results: As a group the patients had significantly shorter mean RT in VS after training $(p<0.001)$ and demonstrated a variety of mechanisms to account for this. Improvements were confined to the training period and maintained at follow up. Three patients had significantly longer RT after training. They had high initial error rates which improved with training. Patients performed ADL tasks significantly faster after training and reported significant subjective improvements. There was no concomitant enlargement of the VF, but there was a small but significant enlargement of the VSF.

Conclusion: Patients can improve VS with practice. This usually involves shorter RTs, but occasionally a longer RT in a complex speed-accuracy trade-off. These changes translate to improved overall visual function, assessed objectively and subjectively, suggesting that they represent robust training effects. The underlying mechanism may involve the adoption of compensatory eye movement strategies.
$\mathrm{P}$ atients with a lesion affecting their posterior visual pathway develop homonymous visual field defects (HVFDs). Such patients are disabled, having particular difficulties with reading and visual exploration that have far reaching repercussions on their domestic and vocational lives. ${ }^{1}$ In the UK at least $50 \%$ of neurological admissions to a general hospital are due to strokes and 30\% of these have HVFDs. Subarachnoid haemorrhages, intracerebral haematomas, and trauma add to this figure. ${ }^{2}$ Rehabilitation strategies for aphasias, motor, and cognitive dysfunction are widely used in clinical practice, ${ }^{3}$ but the rehabilitation of HVFDs has been neglected. ${ }^{4}$

Research into the rehabilitation of patients with brain damage with functional impairments is an intrinsically difficult and laborious task, fraught with methodological problems due to the heterogeneity of the patient group. Despite this, a number of interesting observations have been made regarding hemianopia and the prospect of rehabilitation. First, although lesions of the geniculostriate pathway in man were traditionally considered to result in complete and permanent visual loss in the topographically related area of the visual field (VF) it has become clear that the "blind" hemifields of patients may retain certain visual functions, and can also improve spontaneously. ${ }^{5-7}$

Secondly, patients with field defects intrinsically attempt to compensate for their visual loss. When viewing simple patterns patients with HVFDs paradoxically concentrate their gaze towards their blind hemifield rather than the centre of the pattern ${ }^{8}$; demonstrating what is considered by some to be a compensatory strategy ${ }^{10}$ in that by deviating their fixation point more of the visual scene is brought into their seeing hemifield. Similarly they adopt a number of compensatory strategies when refixating between simple visual targets, which are presented unpredictably. ${ }^{11}$

Thirdly, patients with HVFDs cannot process images in the same way as normal subjects demonstrating numerous refixations and inaccurate saccades that result in disorganised scanning, longer search times and the omission of relevant objects. ${ }^{9}{ }^{12}$ In a previous study we recorded the eye movements of patients with homonymous hemianopia while they viewed images of real scenes. ${ }^{13}$ We found that patients with recent lesions (less than six months old) made patterns of fixations that approximated those of normal controls. Those with more longstanding lesions had increasingly different fixation patterns from those of normal controls. We proposed that this correlation reflected the highly significant evolution of a spontaneous compensatory eye movement strategy, and questioned whether or not the strategy could be manipulated advantageously with specific training in visual search (VS). ${ }^{12}{ }^{14-17}$ There is accumulating evidence that patients can successfully adapt to their HVFD with training in VS. Such training serves to systematically reinforce compensatory oculomotor strategies, thereby fortifying and enlarging their field of search.

In this paper we have devised a novel rehabilitation tool for patients with HVFDs, based on the hypothesis that by training patients in VS they will learn to scan their blind field more efficiently, their ability to search will improve, and

Abbreviations: ADL, activities of daily living; ANOVA, analysis of variance; HVFD, homonymous visual field defect; RT, response time; VF, visual field; VS, visual search; VSF, visual search field 
their disability will reduce. The novel feature of our treatment is that the entire system is portable, low cost and easy to deploy by all therapists. Patients can, therefore, be treated in their own homes or in general rehabilitation environments with minimal disruption to their daily lives under the supervision of personnel who are naive to the technique of VS. We also addressed the question of whether improvements in VS translated to an improvement in their overall visual function, measured objectively in terms of visual tasks representing activities of daily living (ADL) and subjectively with respect to a visual disorder questionnaire.

\section{METHODS}

\section{Patient selection}

A total of 31 patients (16 men, 15 women; mean age 46.9 years, range 24-75) with HVFDs due to a postchiasmatic lesion were recruited and trained in VS. Of these, 28 patients were right handed and three were left handed (Edinburgh handedness inventory ${ }^{18}$ ). Eighteen patients had left and 13 had right field defects, which ranged from homonymous quadrantanopia to complete homonymous hemianopia, with or without macular sparing. Two patients had additional neurological impairments; one had spasmodic torticollis and the other had a stable ipsilateral hemiparesis. Their pathologies were verified with magnetic resonance imaging (MRI) and varied: 22 had cerebral infarctions, six had arteriovenous malformations (AVMs) that had bled, one had a temporal lobectomy, one had a grade 2 glioma in the occipital lobe and one had a suprasellar cystic teratoma. They were recruited at least three months after the onset of their hemianopia, when their field defects were fixed and stable and the confounding effects of spontaneous improvement could be minimised. ${ }^{2}$ Two patients were recruited 3-6 months after onset, three were recruited 6-9 months after onset, five were recruited 912 months after onset, and 21 were recruited $>12$ months after onset. The binocular visual acuities of all patients were corrected to $6 / 6$ (Snellen notation) using trial lenses or contact lenses, because normal corrective spectacle lenses interfered with our eye movement recording equipment. Patients with coexisting eye movement pathology, abnormalities on funduscopy or slit lamp examination, attentional and memory impairments (30 point Mini Mental Test score), ${ }^{19}$ or visual neglect (Rivermead Behavioural Inattention Tests $)^{20}$ were excluded. All work was undertaken with approval from the local ethics committee. All patients gave their informed consent.

\section{Experimental detail}

Training paradigm

Patients were trained in VS on a dedicated $53.34 \mathrm{~cm}$ (21 inch) television monitor, which was installed in their home for the duration of the training and used under supervision. They were encouraged to maximise their concentration by using a quiet darkened room with no visual or auditory distractions. A portable computer was used to generate the VS images on the television monitor. The screen was viewed binocularly at eye level from a distance of $1.5 \mathrm{~m}$, subtending $25^{\circ}$ horizontally by $10^{\circ}$ vertically. This enabled training of the central VF, which is the most valuable to patients exploring their immediate environment.

Several types of VS were created using isor2, a custom made program. Search fields comprising a randomly positioned white target amid white distracter elements were generated on a black background using this software. Target elements were either lines, squares, or triangles and differed from distracters by a single feature, either size (fig lA) or orientation (fig 1B). Prior to the presentation of each search field the patient initially fixated a central cross which was presented for one second to ensure that their fixation

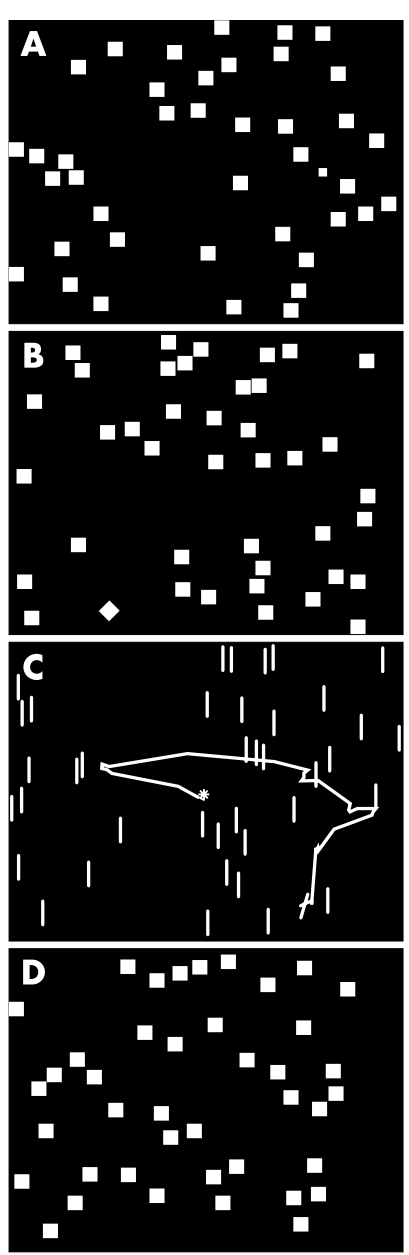

Figure 1 Varieties of visual search task. (A) Square magnification task, where the target square is smaller $\left(1.2^{\circ}\right)$ than the distracters $\left(2^{\circ}\right)$. (B) Square orientation task, where the target square is tilted by $45^{\circ}$ compared with the distracters. (C) Line orientation task with superimposed eye movement trace where the subject initially fixates the central cross $\left({ }^{*}\right)$ and then rapidly searches and fixates the target in the search field. (D) Non-target trial, to determine response accuracy.

commenced at the centre of the screen, and then rapidly searched and fixated the target in the search field which was presented for a maximum of three seconds after the fixation cross had disappeared (fig 1C). Three seconds represents ample time for control subjects to perform VS trials.

Non-target trials constituted $20 \%$ of the total and were randomly interspersed to determine response accuracy (fig 1D). Patients were instructed to press one of the two mouse buttons as soon as they detected a target or had decided one was not present. They were discouraged from making head movements. Each patient received 20 treatment sessions in a one month period, performing 600 trials in target specific blocks of 100 per 40 minute session. Patients were informed of the type of target and distracter prior to each block of trials and were instructed to find the odd one out as quickly as possible. They were not tutored to move their eyes in a suggested pattern, but allowed to develop their own systematic scanning strategies. The response time data were logged on the computer and analysed offline to monitor patients' compliance and progress.

\section{Testing paradigm}

The following assessments were made at each visit: VS response time (RT), ADL tasks, perimetric VFs and visual search fields (VSFs). A subjective visual disorder questionnaire was completed before and after training. An initial visit (visit 0 ) served to familiarise patients with the testing environment and practise the technique of VS. The study design was that of within subject repeated measures and all patients therefore acted as their own controls. Patients were 
tested twice before (visit 1 and 2) and twice after (visit 3 and 4) they were trained in VS. Each visit was one month apart. Changes between visits 1 and 2 reflected spontaneous recovery or practice effects. Changes between visit 2 and 3 represented the true effects of training. The fourth visit was included to determine whether or not the treatment effects were robust and would be maintained beyond the duration of the training.

\section{Visual search}

One of the VS line orientation tasks used to train patients was also used to test them. The search fields were displayed on a high resolution monitor such that they subtended $20^{\circ} \times 20^{\circ}$ at the eye. The apparatus provided a chin rest and clamps at the temples to prevent head movement.

\section{Analysis of response times}

The primary objective in this study was to compare RTs before and after training in VS. To identify errors and inaccuracies, the eye movements made during 200 line orientation VS trials were recorded using the P-scan, a remote video based infrared system. ${ }^{21}$ They were analysed using custom written software. Trials in which errors were made (targets were incorrectly seen/not seen) and those in which a button press was made after the three second viewing time had elapsed (time-out) were excluded. Inaccurate trials, in which patients had shifted their fixation point towards their blind field and the eye movement did not start at the central fixation cross or no fixations fell within $5^{\circ}$ of the target to demonstrate that it had been correctly foveated, were similarly excluded.

The remaining data were analysed to compare RTs for target detection before and after treatment, and between each of the four visits. By considering targets located on the left and right halves of the screen separately we were able to distinguish between RTs to targets presented in the patients' blind and seeing hemifields. A three factor analysis of variance (ANOVA, SPSS version 9), using training period (pre $v$ post-training), visit (four visits, two pre and two posttraining) and field (target located in blind $v$ seeing field) as the factors, was employed to analyse the RTs for each patient individually and as a group. The significance level was set at $\mathrm{p}<0.05$.

\section{Analysis of errors}

Error rates were calculated as the percentage of trials in which targets were incorrectly seen/not seen by patients at each individual visit and were distinguished from trials where patients were timed out.

\section{Assessment of activities of daily living}

Five timed tasks representing activities of daily living were performed at each visit to see whether the search training led to improved ADL performance. The tasks were devised and validated by the authors using a group of 30 control subjects. They were performed by placing various objects in a random distribution on a horizontal board $(1 \times 0.5 \mathrm{~m})$ placed directly in front of the patient. The specific tasks were:

- Coin collection: a selection of UK currency coins were collected one at a time and placed in a small container directly in front of the patient.

- Post box: shapes had to be correctly posted through appropriately shaped holes in posting boxes.

- Bead threading: beads bearing a letter of the alphabet were selected in alphabetical order and threaded onto a leather lace.
- Bead placing: beads bearing a letter of the alphabet were selected in alphabetical order and placed in a row immediately in front of the patient.

- Nut sorting: plumbers' nuts of a particular size were selected from a variety of sizes and stacked on a wooden pillar placed in front of the patient.

Two manual RT control tasks were also performed. Both involved picking up either the nuts or the shapes, used for the ADL tasks described above, from one container and placing them in a second adjacent container. They differed from the ADL tasks in that no searching was required because the objects were directly in front of the patient rather than spread on the board.

A two factor ANOVA with ADL/control task (task 1-5, control 1, control 2) and visit (4 visits, 2 pre and 2 posttraining) as factors was used to analyse the data for each of the ADL tasks individually and as a combined group.

\section{Perimetric assessment of visual fields}

At each visit static, kinetic, and kinetic search fields ${ }^{14}$ were plotted using a Humphrey field analyser under standardised environmental luminance conditions.

\section{Static perimetry}

Static fields were plotted using the Humphrey 30-2 threshold test with automated fixation monitoring.

\section{Kinetic perimetry}

Kinetic fields to $75^{\circ}$ were plotted using the automated Humphrey kinetic program in which a standard I4E target was presented along 16 meridians in a pseudorandom fashion. Target detection was denoted by a button press and the resultant 16 locations were automatically joined to form the I4E isopter. Steady fixation on the central fixation spot was monitored by the experimenter.

\section{Kinetic search fields}

Kinetic search fields were similarly plotted. ${ }^{22}$ An auditory tone signalled the appearance of the target and patients were then encouraged to move their eyes and actively search for it as it moved towards the fixation spot. VFs pre and posttraining were inspected manually and the width of the hemianopic field was measured across the horizontal meridian. A one factor ANOVA was used to determine whether or not the static, kinetic, or kinetic search fields had enlarged with training.

\section{Subjective visual disorder questionnaire}

Patients completed an 11 point self-assessment subjective visual disorder questionnaire, ${ }^{23}$ devised and validated by Kerkhoff et al $^{24}$ before and after training. It was scored using the following 5 point scale: $0=$ no problem, $1=$ rare problem, $2=$ partially relevant problem, $3=$ frequent problem, and $4=$ very frequent problem. Patients were not allowed to review their pretraining answers when completing the posttraining questionnaire. Changes in score for each patient before and after treatment were compared with the MannWhitney rank sum test. Mean scores for each question were compared with Wilcoxon's signed rank test.

\section{RESULTS}

\section{Dropouts}

Of the 31 patients recruited into the study, 29 completed the training and attended all testing sessions. Two patients dropped out; one due to spasmodic torticollis which was aggravated by the requirement to keep her head still, and the other due to social circumstances. 


\section{Analysis of response times and error rates in visual search}

As a group the patients had a significantly shorter mean RT in VS after training (ANOVA $F_{1,28}=14.926, p<0.001$ ) (fig 2). There was no significant spontaneous improvement or practice effect between visits 1 and 2 (the visits prior to the treatment phase). The subsequent improvement in RT with training seen at visit 3 was maintained at visit 4 , a month after the treatment phase had been completed (see fig 2).

When the data for each patient were examined separately with individual ANOVA three distinct groups emerged. Three patients had significantly longer RTs after training, four patients showed no significant change in RTs after training, and the remaining 22 patients had significantly shorter RTs in VS following treatment (ANOVA $\mathrm{p}<0.001$ ). To elucidate the nature of these differences we examined the data further. Most of patients $(76 \%)$ became significantly faster in VS with training. Similar improvements in RT were seen whether the target was located in the blind or the seeing hemifield (fig 3A). Their error rates were less then $10 \%$, and in most cases less than $5 \%$ even prior to training. Four patients (14\%) showed no significant change in RT with training. However, when hemifield effects were considered it became clear that three of these patients became significantly faster at locating targets which fell initially in their blind hemifield and significantly slower at finding targets which fell initially in their seeing hemifield, with no significant overall effect on RT (fig 3B). The fourth patient showed the converse effect and became significantly slower at locating targets which fell initially in his blind hemifield and significantly faster at finding targets which fell initially in his seeing hemifield, with no significant overall effect on RT (fig 3C). Their error rates were less than $5 \%$ even prior to training. Further examination of the three patients who became significantly slower at VS revealed that this effect was observed for targets located in both their blind and seeing hemifields (fig 3D). They were found to have high error rates in excess of $25 \%$ prior to training, which reduced dramatically with treatment. There was a significant inverse correlation between the percentage of errors made prior to training and improvements in RT (Spearman's rank order correlation $\left.R_{29}=-0.481\right)$.

\section{Analysis of tasks representing ADL}

For the patients as a group, improvements in completion times for separate ADL tasks and all ADL tasks collectively were significant (ANOVA $\mathrm{F}_{1,28}=116.590, \mathrm{p}<0.05$ ). There was a trend for each individual patient to perform the ADL tasks faster after training in VS. There were no changes in the completion times for the manual RT control tasks (table 1). We observed a significant correlation between patients' age

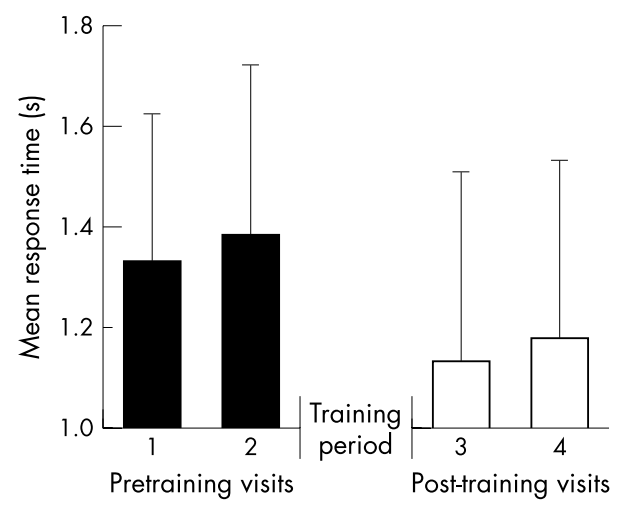

Figure 2 Mean improvement in response time with training for the patients as a group. Error bars $=\mathrm{SD}$.

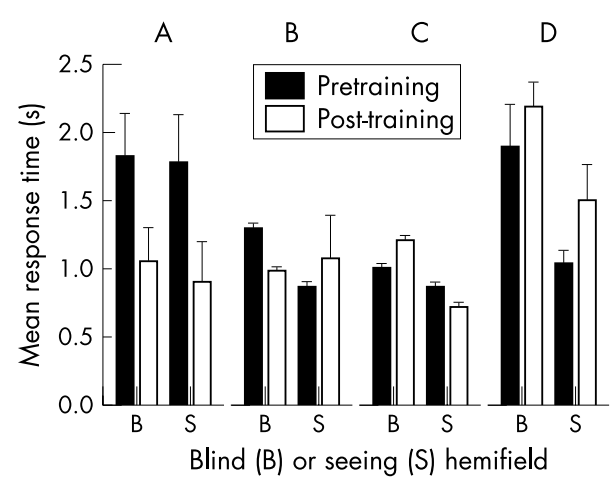

Figure 3 Varieties of training effects highlighting hemifield differences. (A) Similar improvements in response time (RT) irrespective of target location in the blind or seeing hemifield. (B) No significant overall effect on RT, but patients became significantly faster at locating targets in their blind hemifield and significantly slower at finding targets in their seeing hemifield. (C) No significant overall effect on RT, but patient became significantly slower at locating targets in his blind hemifield and significantly faster at finding targets in his seeing hemifield. (D) Similar prolongation of response time irrespective of target location in the blind or seeing hemifield. Error bars $=\mathrm{SD}$.

and magnitude of improvement in the time required to complete the ADL tasks (Spearman rank order correlation $R_{29}=0.402$ ).

\section{Analysis of visual fields}

On inspection of the plots we found no change in the size of the traditional static or kinetic fields of patients after training in VS. There was no expansion in the width of the hemianopic field when measured across the vertical meridian. As a group, there was a small but significant $4^{\circ}$ mean enlargement of their VSF (ANOVA $F_{1,28}=11.517, p<0.05$ ).

\section{Analysis of subjective visual disorder questionnaire}

Complete before and after data were gathered for 27 of the 29 patients. As a group the patients scored significantly better in the subjective questionnaire after training in VS (MannWhitney rank sum test $\mathrm{p}<0.0002$ ). Individually, 19 patients improved their score (reflected by lower score), four patients did not change their score and four patients scored worse (reflected by higher score). When asked directly whether they had noticed a qualitative improvement in their visual function, 19 patients answered affirmatively and eight felt that their vision had not changed. Nevertheless, all patients whose scores remained unchanged or deteriorated claimed to have noticed an improvement.

When the data for each question was considered in turn, there was a trend for improvement in mean scores (reflected by lower score) for each of the 11 questions, but this reached significance for only three (Mann-Whitney rank sum test) (fig 4).

Table 1 Mean task completion time for all ADL tasks and manual response time for tasks 2 and 5, before and after training in visual search

\begin{tabular}{lll}
\hline & \multicolumn{2}{l}{$\begin{array}{l}\text { Mean task completion time in seconds } \\
\text { (SEM) }\end{array}$} \\
\cline { 2 - 3 } Task & Pretraining & Post-training \\
\hline Nut sorting control (task 5) & $18.28(0.81)$ & $18.09(0.74)$ \\
Post box control (task 2) & $34.44(1.19)$ & $34.10(1.20)$ \\
Activities of daily living & $84.88(5.41)$ & $64.01(3.88)$ \\
\hline
\end{tabular}




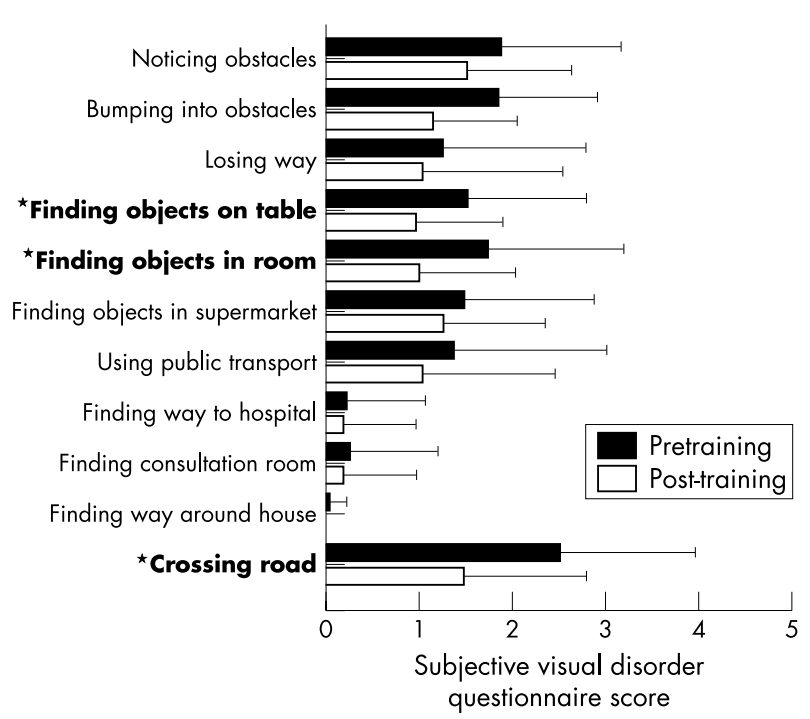

Figure 4 Group results of the subjective visual disorder questionnaire before and after training. 0 , no problem; 5, very frequent problem. Error bars $=S D .{ }^{*} p<0.05$ Mann-Whitney rank sum test.

\section{DISCUSSION}

In the present study we successfully trained 29 patients with HVFD in VS. There were only two dropouts, indicating that the three month programme was well tolerated. We conclude that as a group the patients showed significantly shorter mean RT in VS after training, in the absence of significant spontaneous improvement or practice effects. These improvements appear to be a robust effect of training and were maintained beyond the treatment phase of the study. These data are in keeping with other studies demonstrating that patients with HVFD can become significantly faster in VS with training. ${ }^{12}{ }^{15} 25$ Furthermore, groups which have tried to enlarge the hemianopic field of patients by presenting targets at their field border have also observed significantly faster target detection..$^{26} 27$

However, when the data for each patient were examined individually, three distinct groups emerged. In the first group, which had most of the patients (76\%) the mean values for VS performance improved by $46 \%$ (fig 3A). ${ }^{12} 141527$ In the second group, although four patients showed no significant change in RT with training, the hemifield effects shown in fig 3B, C were demonstrated. We suggest that these represent strategic patterns that have not been highlighted previously. The strategy in fig 3B would involve strengthening the patient's selective attention for the blind hemifield, improving their ability to direct gaze movements towards that side and explore it efficiently. It is possible to interpret the results in fig $3 \mathrm{C}$ as a further strategy in which the patient rapidly scans the intact hemifield before moving on to explore the blind side. In the final group three patients with initial high error rates became significantly slower at VS with training. We suggest that they too showed a more complex improvement despite the fact that their RT lengthened. Before training, they were unable to complete the task in the given time in a high percentage of trials and only responded successfully to salient targets, for example those close to the fixation cross. After training they were able to detect less salient targets within the given time in a complex speed-accuracy trade-off, which accounts for the longer mean RT.

The notion that these findings are strategically based is supported by the observation that there was a trend for improved ADL performance in all 29 patients. Collectively all the patients with hemianopia performed the ADL tasks significantly faster after training by $25 \%$. Interestingly the magnitude of improvement increased with age, suggesting that more elderly patients benefited the most. Since the completion times for the manual RT control tasks did not change following training this suggests that there is no motor practice effect in such tasks. It follows that the ADL tasks were performed faster due to more efficient VS. The question of whether the acquisition of compensatory oculomotor strategies by patients translates to an improvement in their overall visual function rather than being modality specific is of crucial significance. Our study demonstrates this transfer clearly and objectively, in contrast to those which did not control for manual $\mathrm{RT}^{14}{ }^{15}$ and others that only demonstrated a subjective improvement using a questionnaire approach. ${ }^{125}$ In addition, our results show that subjective questionnaires are unreliable. Four of our patients volunteered a qualitative improvement in their visual function with training in VS, but their actual ratings deteriorated. We suggest that the period of training serves to make patients aware of the full extent of their visual handicap, causing them to internally re-evaluate their own visual loss. In fact, several patients commented that their vision appeared to deteriorate initially and then improve as training progressed. The post-training questionnaire was therefore completed with a more profound understanding of their hemianopia.

The small $\left(4^{\circ}\right)$ but significant enlargement in the group mean VSF provides further evidence to support the notion that they were able to search better after training. VSFs were originally conceptualised by Kerkhoff as "the area in the perimeter that a patient could scan via eye movements, but without head movements, when searching for a bright suprathreshold stimulus". ${ }^{14}$ They were readily adopted by subsequent groups as a measure of a patient's capacity for effective VS. None of our patients demonstrated the dramatic enlargements of $20^{\circ}$ or $30^{\circ}$, recorded in other series. ${ }^{14}{ }^{15}$

With respect to VF changes, we found no significant enlargement of the static or kinetic VF with training. In contrast with others, we preferred to use automated kinetic perimetry rather than standard Goldmann perimetry because the results are entirely objective and investigator bias is eliminated. The potential for recovery of the lost VF has sparked a significant conflict of opinion, which is ongoing. Training techniques have used either VS or a different approach involving repetitive stimulation of the transition zone between the intact and the damaged fields and VF enlargements have been observed by some groups ${ }^{12} 14$ 15 28-30 but not by others. ${ }^{31-33}$ With our experimental design, we were unable to restore the lost VF, but accept that restitution training may require substantially more training than that required to adopt compensatory oculomotor strategies. In any event, enlargement of the VF or VSF does not imply normal scanning. ${ }^{27}$

In clinical practice strokes are commonplace and many such patients have VF defects which cause a functional disability that impinges on all other aspects of their rehabilitation. Given the importance of vision as a special sense, the treatment of hemianopia should assume a priority role for neurological rehabilitation programmes. In practice, however, this has not been the case and to date in the UK research into this area of rehabilitation is notable by its absence. We have presented a simple, inexpensive, and effective retraining method. It is now necessary to address the specificity of the technique in order to establish its success. A randomised controlled trial including patients who are not trained therefore needs to be undertaken. However, in response to clinical need the technique is now available on videotape and therefore accessible to almost all patients, since even those who are technically unsophisticated own televisions and video players. 


\section{Authors' affiliations}

A L M Pambakian, S K Mannan, T L Hodgson, C Kennard, Department of Sensory Motor Systems, Division of Neuroscience and Psychological Medicine, Imperial College School of Medicine, London, UK

We gratefully acknowledge the support of the Stroke Association (5/97) and the Wellcome Trust (037222) in funding this research.

Competing interests: none declared

\section{REFERENCES}

1 Hallett PE, Lightstone AD. Saccadic eye movements to flashed targets. Vision Res 1976;16:107-14.

2 Pambakian AL, Kennard C. Can visual function be restored in patients with homonymous hemianopia? Br J Ophthalmol 1997;81:324-8.

3 Neurological Rehabilitation. London: Churchill Livingstone, 1993

4 Zihl J. Rehabilitation of visual disorders after brain injury. Hove: Psychology Press, 2000.

5 Poppelreuter W. Die Störungen der niederen und höreren Sehleistungen durch Verletzungen des Okzipitalhirns. In: Die psychischen Schädigungen durch Kopfschuß im Kriege 1914/16. Leipzig: Leopold Voss, 1917.

6 Riddoch G. Dissociation of visual perceptions due to occipital injuries, with especial reference to appreciation of movement. Brain 1917:40:15-57.

7 Hine $M$. The recovery of fields of vision in concussion injuries of the occipital cortex. Br J Ophthalmol 1918;2:12-25.

8 Ishiai S, Odajima N, Kotera M, et al. [Eye fixation-patterns in homonymous hemianopia and unilateral spatial neglect: a study with an eye camera] Rinsho Shinkeigaku 1987:27:634-41.

9 Chedru F, Leblanc M, Lhermitte F. Visual searching in normal and braindamaged subjects (contribution to the study of unilateral inattention). Cortex 1973;9:94-111

10 Gassel MM, Williams D. Visual function in patients with homonymous hemianopia. Part II. Oculomotor mechanisms. Brain 1963:86:1-36.

11 Meienberg $\mathrm{O}$, Zangemeister WH, Rosenberg M, et al. Saccadic eye movement strategies in patients with homonymous hemianopia. Ann Neurol 1981;9:537-44.

12 Zihl J. Visual scanning behavior in patients with homonymous hemianopia. Neuropsychologia 1995:33:287-303.

13 Pambakian AL, Wooding DS, Patel N, et al. Scanning the visual world: a study of patients with homonymous hemianopia. I Neurol Neurosurg Psychiatry 2000;69:751-9.

14 Kerkhoff G, Münssinger U, Haaf E, et al. Rehabilitation of homonymous scotomata in patients with postgeniculate damage of the visual system: saccadic compensation training. Restor Neurol Neurosci 1992;4:245-54.
15 Kerkhoff G, Munssinger U, Meier EK. Neurovisual rehabilitation in cerebral blindness. Arch Neurol 1994;51:474-81.

16 Zihl J. [Homonymous hemianopsia and its rehabilitation]. Klin Monatsb/ Augenheilkd 1988;192:555-8.

17 Nelles G, Esser J, Eckstein A, et al. Compensatory visual field training for patients with hemianopia after stroke. Neurosci Lett 2001;306:189-92.

18 Oldfield RC. The assessment and analysis of handedness: the Edinburgh inventory. Neuropsychologia 1971;9:97-113.

19 Zeki S. A century of cerebral achromatopsia. Brain 1990;113(Pt 6):1721-77.

20 Wilson B, Cockburn J, Halligan P. Development of a behavioral test of visuospatial neglect. Arch Phys Med Rehabil 1987;68:98-102.

21 Barbur JL, Thompson WD, Forsyth PM. A new system for the simultaneous measurement of pupil size and two-dimensional eye-movements. Clin Vis Sci 1987; 2:131-42.

22 Kerkhoff G, Münssinger U, Haaf E, et al. Rehabilitation of homonymous scotomata in patients with postgeniculate damage of the visual system: saccadic compensation training. Restor Neurol Neurosci 1992:4:245-54.

23 Nelles G, Esser J, Eckstein A, et al. Compensatory visual field training for patients with hemianopia after stroke. Neurosci Lett 2001;306 189-92.

24 Kerkhoff G, Schaub J, Zihl J. [Anamnesis of brain-originated vision disorders]. Nervenarzt 1990;61:711-18.

25 Poggel DA, Kasten E, Muller-Oehring EM, et al. Unusual spontaneous and training induced visual field recovery in a patient with a gunshot lesion. J Neurol Neurosurg Psychiatry 2001;70:236-9.

26 Kasten E, Wust S, Behrens-Baumann W, et al. Computer-based training for the treatment of partial blindness. Nat Med 1998;4:1083-7.

27 Kasten E, Poggel DA, Sabel BA. Computer-based training of stimulus detection improves color and simple pattern recognition in the defective field of hemianopic subjects. J Cogn Neurosci 2000;12:1001-12.

28 Savir H, Michelson I, David C, et al. Homonymous hemianopsia and rehabilitation in fifteen cases of C.C.I. Scand J Rehabil Med 1977;9:151-3.

29 Zangemeister WH, Meienberg O, Stark L, et al. Eye-head coordination in homonymous hemianopia. J Neurol 1982;226:243-54.

30 Kasten E, Sabel BA. Visual-field enlargement after computer-training in braindamaged patients with homonymous deficits-an open pilot trial. Restor Neurol Neurosci 1995;8:113-27.

31 Bach-Y-Rita P. Controlling variables eliminates hemianopsia rehabilitation results. Behav Brain Sci 1983;6:448.

32 Balliet R, Blood KM, Rita P. Visual field rehabilitation in the cortically blind? J Neurol Neurosurg Psychiatry 1985;48:1113-24.

33 Pommerenke K, Markowitsch HJ. Rehabilitation training of homonymous visual field defects in patients with postgeniculate damage of the visual system. Restor Neurol Neurosci 1989;1:47-63. 